\title{
Multi-scale local pattern co-occurrence matrix for textural image classification
}

\author{
Xiangping Sun*, Jin Wang, Ronghua Chen, Mary F.H. She, Lingxue Kong \\ The Institute for Technology Research and Innovation \\ Deakin University \\ Waurn Ponds, Vic, 3216, Australia \\ \{Sxian, wjin, ronghuac, mary.she, lingxue.kong\}@deakin.edu.au
}

\begin{abstract}
Textural image classification technologies have been extensively explored and widely applied in many areas. It is advantageous to combine both the occurrence and spatial distribution of local patterns to describe a texture. However, most existing state-of-the-art approaches for textural image classification only employ the occurrence histogram of local patterns to describe textures, without considering their cooccurrence information. And they are usually very timeconsuming because of the vector quantization involved. Moreover, those feature extraction paradigms are implemented at a single scale. In this paper we propose a novel multi-scale local pattern co-occurrence matrix (MS_LPCM) descriptor to characterize textural images through four major steps. Firstly, Gaussian filtering pyramid preprocessing is employed to obtain multi-scale images; secondly, a local binary pattern (LBP) operator is applied on each textural image to create a LBP image; thirdly, the gray-level co-occurrence matrix (GLCM) is utilized to extract local pattern co-occurrence matrix (LPCM) from LBP images as the features; finally, all LPCM features from the same textural image at different scales are concatenated as the final feature vectors for classification. The experimental results on three benchmark databases in this study have shown a higher classification accuracy and lower computing cost as compared with other state-of-the-art algorithms.
\end{abstract}

Keywords-local pattern; co-occurrence; multi-scale; vector quantization; LPCM

\section{INTRODUCTION}

Textural image classification has been widely applied in many areas including medical image analysis, remote sensing, material characterization, and content-based image retrieval (CBIR). However, the development of effective and efficient textural image classification approach remains a challenging task due to the existence of large intra-class variation and inter-class similarity in textural images [1].

Though there is no unanimous definition, texture is usually described as a combination of characteristics, including uniformity, density, coarseness, roughness, regularity, and intensity [2, 3]. In the textural images these features can be depicted by the intensity and spatial arrangement of pixels [4]. In addition, textures can occur at arbitrary spatial resolution and orientation in the real world. Thus, denoting the smallest unit of a texture as a local pattern, considering both the occurrence and spatial distribution of local patterns at multiple scales should be advantageous in order to effectively characterize textures in an image.
Most existing state-of-the-art methods for textural image classification are statistics-based. The representative statistical approaches include the filter bank-based methods $[1,5,6]$, patch-based algorithms [7, 8], bags-of-keypoints [9-12]. The filter bank-based methods retrieve the textural image feature from the responses of a bank of filters. In fact, no matter which filters used - LM filter bank [5], S filter bank [6] or MR8 filter bank [1], the filter response at each image pixel could all be regarded as a local pattern that characterizes the small neighborhood of that pixel. And the occurrence histogram of the quantized local patterns was adopted as the final feature in those approaches. The patch-based methods utilize raw pixel values in image patches for classification. Here the local patch becomes the local pattern, and the occurrence frequency of the quantized local patterns is also utilized as the image feature. However, as the patch-based methods use the row reordered vector from the raw image data as local pattern, they are not robust to noise, non-linear intensity change, and rotation. In the bag-of-keypoints approaches, the image descriptors such as SIFT [13] and HoG [14] are utilized to extract features from the detected interest areas of images as the local patterns, and then the number of these sparsely-distributed local patterns is recorded as final image feature. The bag-of-keypoints methods work very well in object image classification tasks. However, they are not suitable for the textural image classification since most textures are uniform, and thus it is difficult to detect any representative keypoints and interest areas from them.

Regardless of the popularity of these three approaches, there are two common problems with them: (1) only the occurrence of the local patterns is considered in the image feature, and the global spatial distribution of them, or the cooccurrence is ignored; (2) since a large amount of computation is involved in the vector quantization these methods are very time-consuming, especially for the filter bank and patch-based methods in which the number of local patterns equals the number of the pixels in images.

In a recent work, Yang and Newsam [15] proposed a visual words-based co-occurrence method to extract spatial information between local patterns. A co-occurrence matrix of the local patterns was constructed as the feature vector. To reduce the size of the co-occurrence matrix, a small-size codebook was learned to construct the co-occurrence matrix. Since this co-occurrence based feature is not very representative, another large-size codebook was also learned to represent the images as a bag of words, and combined these two features together as the image feature vector. This implies 
that the vector quantization step will be repeated twice, which is even more computationally expensive.

To tackle these problems, in this paper we incorporate the co-occurrence information of the local patterns into the image feature, instead of conventional gray-level co-occurrence matrix (GLCM) [16]. Unlike GLCM which records the occurrence number of a complete set of intensity pixel pairs in a gray-level image, we use a local pattern co-occurrence matrix (LPCM) descriptor to count the number of local pattern pairs subject to a specific relationship in a local pattern image. The local binary pattern (LBP) operator [17] is utilized to retrieve local patterns from the images. The LBP serves as a texture operator that encodes the local neighborhoods of each pixel in an image as a LBP code, which has proved to be very efficient to represent textures elsewhere. A prominent advantage of the LBP is that, the LBP codes could be directly used as the code words, and no vector quantization is needed since they are integers. Thus it is very time-efficient. Furthermore, the parameters of the LBP operator could be changed to describe multi-scale neighborhood. Because of the flexibility of the LBP operator, many LBP variants have been developed to endow it with the rotation invariance property and more discriminative power [17]. Since the co-occurrence matrix of the local patterns contains both the occurrence and co-occurrence information (the sum of the co-occurrence number in the ith row equals the occurrence number of the ith pattern approximately), we utilize it directly as the feature vector. By utilizing the rotation invariant uniform $\mathrm{LBP}\left(\mathrm{LBP}_{\mathrm{P}, \mathrm{R}}^{\mathrm{riu} 2}\right)$ operator to extract the local patterns, the dictionary size is very small, thus the size of the co-occurrence matrix constructed would not be too large.

In addition, since the LPCM descriptor still just considers the local spatial information, to fully explore global spatial information of textural images, a multi-scale technique - the Gaussian pyramids - is applied to extract the spatial information of local patterns in different scales of the images, i.e., multi-scale LPCM descriptor.

In this paper we apply the proposed method to classify the textural images in six datasets retrieved from three benchmark databases by utilizing the chi-square kernel SVM as the classifier. Experimental results show that our method outperforms the state-of-the-art algorithms in various aspects.

The rest of the paper is organized as follows. In Section 2 we will give a brief introduction of LBP and GLCM. The multi-scale local pattern co-occurrence matrix algorithm is presented in Section 3. We experimentally evaluated the method in Section 4, in comparison to a few state-of-the-art algorithms for textural image classification on several benchmark datasets. In the last section we briefly conclude the major contributions of the work.

\section{RELATED WORKS}

In this section we introduce the LBP operator and some of its variants, and the GLCM method.

\subsection{The LBP operator}

LBP [17] is a texture operator that characterizes the microstructures of an image. Considering a circularly symmetric neighborhood with radius $R$ where $P$ pixels are equally located on the circle, the LBP operator translates the joint distribution of these $\mathrm{P}$ pixels with the centre pixel $\mathrm{C}$ into a binary pattern, which is further encoded as a LBP code (an integer). It is defined as:

$$
\mathrm{LBP}_{\mathrm{P}, \mathrm{R}}=\sum_{\mathrm{p}=0}^{\mathrm{P}-1} \mathrm{~s}\left(\mathrm{~g}_{\mathrm{p}}-\mathrm{g}_{\mathrm{C}}\right) 2^{\mathrm{p}}
$$

where $\left\{\mathrm{g}_{0}, \ldots, \mathrm{g}_{\mathrm{P}-1}\right\}$ and $\mathrm{g}_{\mathrm{C}}$ are the intensities of the $P$ neighboring pixels and the center pixel $C$, respectively, and

$$
s\left(g_{p}-g_{C}\right)= \begin{cases}1, & \text { if } g_{p} \geq g_{C} \\ 0, & \text { otherwise }\end{cases}
$$

An example of the LBP code calculation from an 8neighborhood of a pixel can be seen in Figure 1.
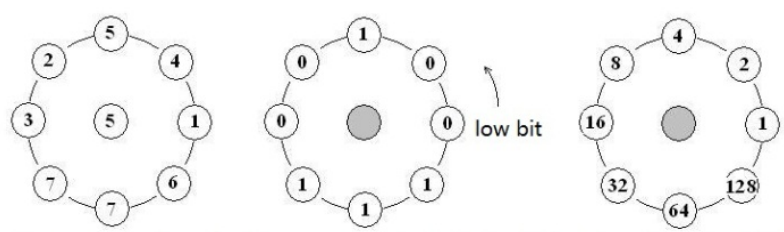

the 8-neighborhood binary pattern: 00100111 the LBP code: $4+32+64+128=228$

Figure 1. Calculating the LBP code from a 8- neighborhood

Considering that this original LBP operator is not invariant to the image rotation, a rotation invariant LBP, i.e., $\mathrm{LBP}^{\mathrm{ri}}$ is proposed in [17], where the $\mathrm{LBP}^{\mathrm{ri}}$ code is assigned as the smallest LBP code of all the binary patterns that are created by circularly shifting the original binary pattern. The LBP ${ }^{\text {ri }}$ operator is defined as:

$$
\operatorname{LBP}_{\mathrm{P}, \mathrm{R}}^{\mathrm{ri}}=\min \left\{\operatorname{ROR}\left(\operatorname{LBP}_{\mathrm{P}, \mathrm{R}}, \mathrm{i}\right), \mathrm{i}=0,1, \ldots, \mathrm{P}-1\right\}
$$

where $\operatorname{ROR}\left(\mathrm{LBP}_{\mathrm{P}, \mathrm{R}}, \mathrm{i}\right)$ performs the right shift of the binary pattern $\mathrm{LBP}_{\mathrm{P}, \mathrm{R}}$ by $i$ bits.

Furthermore, empirical observations show that in textures only certain local binary patterns account for the fundamental properties, and others are insignificant. Thus a rotationinvariant uniform LBP operator LBP ${ }^{\text {riu2 }}$ was developed [17], in which the fundamental patterns are selected through a 'uniform' measurement. In the LBP ${ }^{\text {riu2 }}$ operator, the fundamental patterns are regarded as 'uniform', while all others are encoded as one 'non-uniform' pattern. The LBPriu2 operator is defined as:

$$
\mathrm{LBP}_{\mathrm{P}, \mathrm{R}}^{\text {riu2 }}=\left\{\begin{array}{cc}
\sum_{\mathrm{p}=0}^{\mathrm{P}-1} \mathrm{~s}\left(\mathrm{~g}_{\mathrm{p}}-\mathrm{g}_{\mathrm{C}}\right) & \text { if } \mathrm{U}\left(\mathrm{LBP}_{\mathrm{P}, \mathrm{R}}\right) \leq 2 \\
\mathrm{P}+1 & \text { otherwise }
\end{array}\right.
$$

where

$$
\begin{aligned}
& \mathrm{U}\left(\mathrm{LBP}_{\mathrm{P}, \mathrm{R}}\right)=\left|\mathrm{s}\left(\mathrm{g}_{0}-\mathrm{g}_{\mathrm{C}}\right)-\mathrm{s}\left(\mathrm{g}_{\mathrm{P}-1}-\mathrm{g}_{\mathrm{C}}\right)\right| \\
& +\sum_{p=1}^{P-1}\left|s\left(g_{p}-g_{C}\right)-s\left(g_{p-1}-g_{C}\right)\right|
\end{aligned}
$$

From $\operatorname{LBP}_{\mathrm{P}, \mathrm{R}}$ to $\mathrm{LBP}_{\mathrm{P}, \mathrm{R}}^{\mathrm{riu} 2}$, the sizes of the codebooks constructed decreases from $2^{\mathrm{P}}$ to $\mathrm{P}+2$, while on the contrary the discriminative power increases. In addition, through changing of the parameters $R$ and $P$ of the LBP operator, different scales and details of the microstructures of images 
could be characterized. Three common sets of parameters are: $\{(\mathrm{R}, \mathrm{P})=(1,8),(2,16),(3,24)\}$.

\subsection{Gray-level co-occurrence matrix}

The gray-level co-occurrence matrix (GLCM) [16] is a method that measures the co-occurrence frequency of pixelpairs with all possible combinations of intensities subject to some specific spatial relationship. Denoting each element in GLCM matrix as $P_{d, \alpha}\left(g, g^{\prime}\right)$, where g, $g^{\prime} \in$ \{gray levels in the image , it counts the number of pixel-pairs in the image that have intensity values of $g$ and $g$ ' and are separated by a pixel distance $d$ in direction $\alpha$ (Figure 2).

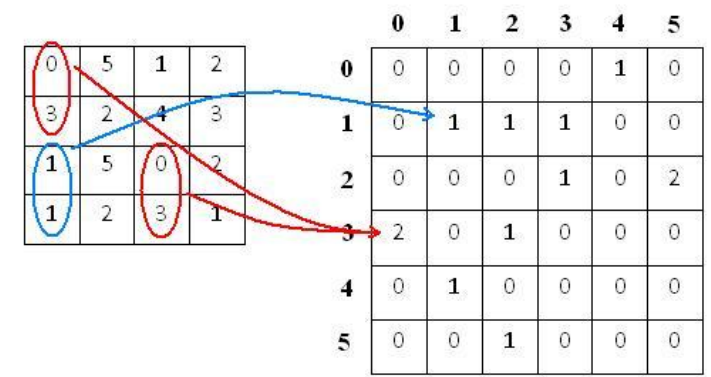

Figure 2. An example of the GLCM matrix construction from a small image, where $d=1, \alpha=90^{\circ}$, and the gray levels range from 0 to 5 .

Since the GLCM is very large and sparse (for an 8-bit graylevel image, the number of gray levels is 256 , and so the size of a GLCM is $256^{*} 256$ ), it could not be directly used as image feature. Some techniques such as gray-level quantization are applied to reduce e matrix dimension. Some feature extraction methods have been utilized to extract more effective image features from the matrix, instead of directly using it [16]. But these approaches have some drawbacks as the gray-level quantization will lose a large number of detailed information, while the features extracted from the GLCM matrix are not discriminative. However, it is anticipated that this problem won't exist in the co-occurrence matrix construction from LBP images, since the LBP codebook size can be very small (using the $L B P_{P, R}^{r i u 2}$ operator). Thus the constructed LPCM could be directly used as image feature, which will greatly improve the discriminative power of the LBP operator.

\section{MULTI-SCALE LOCAL PATTERN CO-OCCURRENCE MATRIX (MS_LPCM) FOR TEXTURE FEATURE EXTACTION}

As discussed in Section 1, both of the occurrence and spatial distribution of local patterns are important to the characterization of texture features present in images. Unlike most of the existing statistical texture feature extraction methods which only take into account the occurrence frequency of the local patterns while ignoring the spatial distribution, we extend the standard LBP descriptor or its variants to enable more effective and efficient texture feature extraction by taking the advantages of both the robustness of the LBP operator to represent the local neighborhood and the ability of the GLCM method to extract spatial information between the local binary patterns. Specifically, after multiscale Gaussian filtering pre-processing in a pyramid fashion, the GLCM matrices constructed from the LBP images at different scales are directly concatenated as the image feature for classification by a Support Vector Machine (SVM). The flowchart of the proposed method is shown in Figure 3.

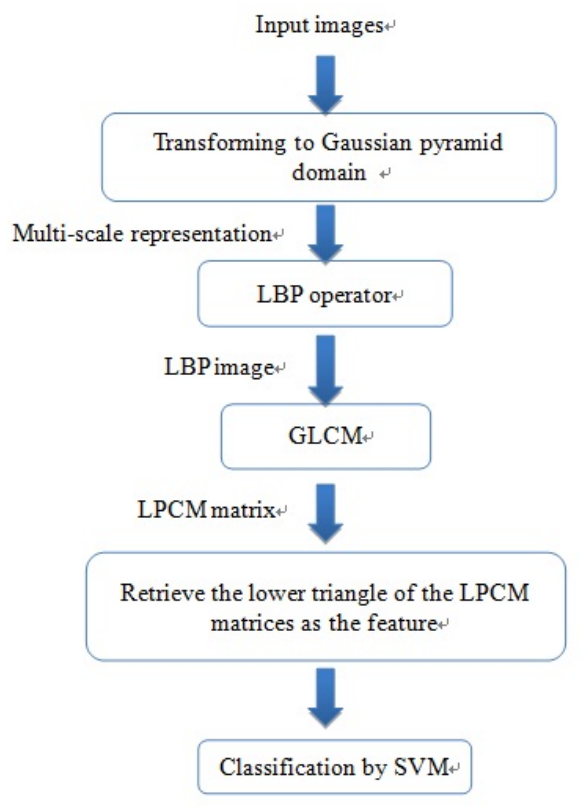

Figure 3. The flowchart of the multi-scale local pattern co-occurrence matrix (LPCM) based textural image classification

\subsection{The LPCM descriptor}

The LPCM descriptor generates a co-occurrence matrix from the created local pattern image as textural image feature. Here the local pattern image is constructed by the LBP codes through applying the LBP operator on a local neighborhood of each pixel in the texture images. Due to the gray-scale invariance inherited from the standard LBP, the LPCM descriptor is also invariant to the gray-scale change. On the other hand, although the LBP is rotation invariant, the LPCM descriptor might not be robust to the rotation variations, since the GLCM is not invariant to the rotation. To endow the rotation invariant property to the LPCM descriptor, we generate several GLCM matrices in the evenly distributed orientations along the circle from a local binary pattern image, and then sum these matrices together as the LPCM feature:

$$
\begin{gathered}
\operatorname{LPCM}_{\mathrm{d}, \mathrm{N}, \mathrm{P}, \mathrm{R}} \text { (image i) }= \\
\sum_{\mathrm{j}=0}^{2 \mathrm{~N}-1} \mathrm{GLCM}_{\mathrm{d}, \mathrm{j} * \Delta}\left(\operatorname{LBP}_{\mathrm{P}, \mathrm{R}}^{\text {riu2 }}(\text { image i })\right)
\end{gathered}
$$

where $\Delta=\pi / \mathrm{N}$ and $\alpha=\{\mathrm{j} * \Delta, \mathrm{j}=0, \ldots, 2 \mathrm{~N}-1\}, 2 N$ is the number of orientations considered. Here the rotation invariant uniform LBP operator is utilized for higher discriminative power and lower size of dictionary constructed. By utilizing the symmetric GLCM representation, Equation (4) could be rewritten as:

$$
\begin{gathered}
\operatorname{LPCM}_{\mathrm{d}, \mathrm{N}, \mathrm{P}, \mathrm{R}}(\text { image } \mathrm{i})= \\
\sum_{\mathrm{i}=0}^{\mathrm{N}-1} \operatorname{symmetricGLCM}_{\mathrm{d}, \mathrm{j} * \Delta}\left(\operatorname{LBP}_{\mathrm{P}, \mathrm{R}}^{\text {riu2 }}(\text { image i })\right)
\end{gathered}
$$

Considering that the LPCM matrix is symmetric, we retrieve the lower triangle as the image feature, as shown in Figure 4. 


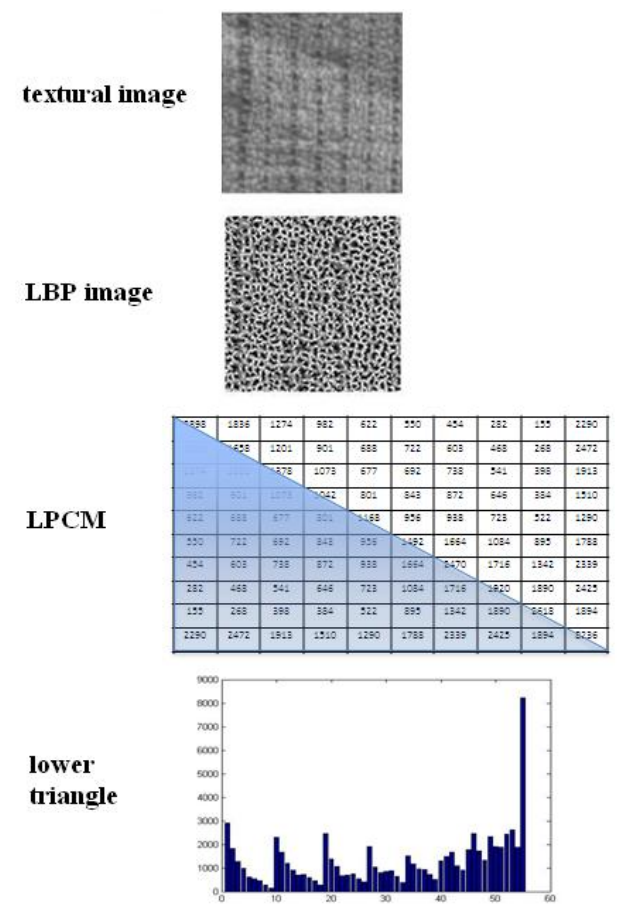

Figure 4. Feature extraction using the LPCM descriptor

For the four parameters of LPCM - (d, N, P, R), $d$ and $R$ could be changed to characterize the local neighborhoods with different sizes (the radius of the local neighborhood LPCM describes is $\mathrm{d}+\mathrm{R}$ ), while $N$ and $P$ determine the complexity of the descriptor. Since the size of the LBP code dictionary should be kept small, the local neighborhood should be small as well; otherwise the LBP codes might lose their representativeness. In this paper we fix the complexity of the LPCM descriptor by setting $P=8, N=4$, and vary the scale parameters as $d=\{1,2$, $3\}, \mathrm{R}=\{1,2,3\}$, respectively.

\subsection{Multi-scale representation in Gaussian pyramid domain}

Since the LBP operator only characterizes the spatial distribution of pixels in a local neighborhood, and GLCM describes that of the local pattern-pairs at a certain short distance with weak relationship between local patterns far away from each other, the LPCM descriptor could only capture local spatial information. In order to overcome those drawbacks by capturing spatial information at larger areas, the Gaussian pyramid technique is applied on the textural images to realize multi-scale analysis, as shown in Figure 5. Then the LPCM features are extracted from all images in the Gaussian pyramid domain and concatenated as final feature vector for textural image classification. The Gaussian filter is defined as:

$\mathrm{G}(\mathrm{x}, \mathrm{y}, \sigma)=\frac{1}{2 \pi \sigma^{2}} \mathrm{e}^{-\left(\mathrm{x}^{2}+\mathrm{y}^{2}\right) / 2 \sigma^{2}}$

According to the setting of $\sigma$ in [13], we set $\sigma=1.5$ here. And we set the level of the Gaussian pyramid as 5 .

\subsection{Kernel SVM}

To classify textural images we use the kernel Support Vector Machine (SVM) [18]. For the two-class case, the SVM constructs a hyper-plane to separate the data-points belonging to them by solving the following optimization problem:

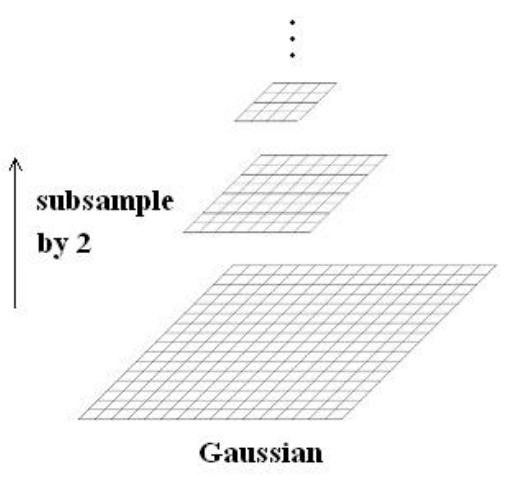

Figure 5. The Gaussian scale space

$$
\begin{gathered}
\min _{\beta, \beta_{0}} \frac{1}{2}\|\beta\|^{2}+C \sum_{\mathrm{i}=1}^{\mathrm{N}} \varepsilon_{\mathrm{i}} \\
\text { s.t. } \varepsilon_{\mathrm{i}} \geq 0, \mathrm{y}_{\mathrm{i}}\left(\mathrm{h}\left(\mathrm{x}_{\mathrm{i}}\right)^{\mathrm{T}} \beta+\beta_{0}\right) \geq 1-\varepsilon_{\mathrm{i}} \forall \mathrm{i},
\end{gathered}
$$

where $\mathrm{h}\left(\mathrm{x}_{\mathrm{i}}\right)=\left\{\mathrm{h}_{1}\left(\mathrm{x}_{\mathrm{i}}\right), \mathrm{h}_{2}\left(\mathrm{x}_{\mathrm{i}}\right), \ldots, \mathrm{h}_{\mathrm{M}}\left(\mathrm{x}_{\mathrm{i}}\right)\right\}$ is a set of basis functions which map the input vector $x_{i}$ into a higher dimensional space.

The decision function for a test sample $\mathrm{x}$ is:

$$
g(x)=\sum_{i} \alpha_{i} y_{i} K\left(x_{i}, x\right)+\beta_{0}
$$

where $\alpha_{\mathrm{i}}$ is a non-zero coefficient of the training sample $\left(\mathrm{x}_{\mathrm{i}}, \mathrm{y}_{\mathrm{i}}\right)$, and $\beta_{0}$ is the parameter of the hyper-plane, which could both be learned by solving Equation $(7) . K\left(x_{i}, x\right)=$ $\left\langle\mathrm{h}\left(\mathrm{x}_{\mathrm{i}}\right), \mathrm{h}(\mathrm{x})\right\rangle$ is the kernel function which computes the inner product of two vectors $\mathrm{x}_{\mathrm{i}}$ and $\mathrm{x}$ in the transformed space.

In the multi-class case, the SVM creates a hyper-plane between every two different classes. And also for each pair of classes a decision function will be learnt.

Because of the effectiveness of chi-square distance in histogram based image classification, we incorporate it into the SVM as the chi-square kernel [19], which is defined as:

$$
\mathrm{K}(\mathrm{x}, \mathrm{y})=1-\frac{1}{2} \sum_{\mathrm{k}=1}^{\mathrm{K}} \frac{\left(\mathrm{x}_{\mathrm{k}}-\mathrm{y}_{\mathrm{k}}\right)^{2}}{\left(\mathrm{x}_{\mathrm{k}}+\mathrm{y}_{\mathrm{k}}\right)}
$$

where $K$ is the dimension of the vectors $x$ and $y$.

In this paper we adopt a one-against-one strategy, and train a SVM classifier between each pair of the classes for multiclass classification. To implement SVM we refer to the libSVM software [20].

\section{EXPERIMENTAL EVALUATION}

To evaluate the effectiveness of our method, we tested it on six datasets selected from three benchmark texture databases: Broadtz [21], CURet [22], and OUTex [23], in comparison to some state-of-the-art methods for texture classification.

\subsection{Methods in comparison}

Four representative state-of-the-art methods have been selected to compare with our approach with respect to both the classification accuracy on the six datasets and the computation complexity. The first one is $\operatorname{LBP}_{\mathrm{P}, \mathrm{R}}^{\mathrm{riu} 2}[17]-$ a rotation 
invariant uniform local binary pattern descriptor. It is chosen as our rival for two reasons: firstly, the proposed method is based on the $\mathrm{LBP}_{\mathrm{P}, \mathrm{R}}^{\text {riu2 }}$ operator; secondly, the $\mathrm{LBP}_{\mathrm{P}, \mathrm{R}}^{\mathrm{riu} 2}$ operator is very powerful and widely used in describing the texture. In the experiments we will evaluate the $\mathrm{LBP}_{\mathrm{P}, \mathrm{R}}^{\mathrm{riu} 2}$ at multi scales by setting $(P, R)=\{(8,1),(16,2),(24,3)\}$.

The second approach is VZ_MR8 [1] which is the most popular filter bank-based method in the literature. MR8 is a filter bank that consists of 36 directional filters (an edge filter and a bar filter, at 6 orientations and 3 scales each), a Gaussian filter and a Laplacian of Gaussian filter. The maximum filter responses at the six different orientations, together with the responses from the Gaussian filter and Laplacian of Gaussian filter, constitute the 8 outputs for the VZ_MR8 discriptor.

The third method VZ_Joint [7] is patch based. It uses raw image data as local pattern, and has been demonstrated with very good performance on some benchmark texture datasets.

The fourth one is another multi-scale algorithm for texture classification - MS-BP [24]. This approach utilized Gaussian pyramid technique to generate a multi-scale basis for each image, and then applied four filters to extract the multistructures from multi-scale images. LBP is finally employed to retrieve image feature from the filtered images.

Furthermore, we will also give a comparison between our results and the best classification accuracies achieved on these datasets so far.

\subsection{Experimental setups}

We selected 6 popular texture classification test suites from the three databases to evaluate the robustness of the proposed method on characterizing different textural images.

Brodatz 24-class dataset $\left(\right.$ Brodat $\left.^{24}\right)$ : There are 24 textural images in this dataset, where each image is divided into $5 \times 5$ non-overlapping subimages. 13 subimages are randomly selected for training, while the rest 12 are used for testing.

Broadtz full dataset (Brodatz ${ }^{\text {Full }}$ ): All 111 textural images in the Brodatz database are utilized. While each image is partitioned into $3 \times 3$ non-overlapping subimages, 3 of them are used for training and the other 6 are for testing. The challenge of this dataset lies in the large number of texture classes and the small number of samples within each class.

CURet dataset: This dataset contains 61 classes of textures, where each class of texture is composed of 92 images captured with varying viewing and illumination parameters. According to the setup in [1], we randomly selected 46 samples from each class for training, and used the rest 46 for testing. This dataset is useful for evaluating the viewpoint and illumination invariance property of the proposed method.

$$
\begin{aligned}
& \text { OUTex_TC_00010 dataset }\left(\mathrm{OUTex}^{10}\right) \text { : } \\
& \text { OUTex_TC_00012 ('tl84') dataset (OUTex } \left.{ }^{12-\mathrm{t}}\right) \text { : } \\
& \text { OUTex_TC_00012 ('horizon') dataset (OUTex }{ }^{12 \_h} \text { ): }
\end{aligned}
$$

These three datasets have the same 24 classes of textures. Each texture is captured in 9 rotation angles $\left\{0^{\circ}, 5^{\circ}, 10^{\circ}, 15^{\circ}\right.$, $\left.30^{\circ}, 45^{\circ}, 60^{\circ}, 75^{\circ}, 90^{\circ}\right\}$, and 20 sample images in each angle.
In OUTex_TC_00010 all the textures are imaged under the 'inca' illuminant. While in OUTex_TC_00012 two other illuminants ('tl84', 'horizon') are used. In the experiments, 480 images from OUTex_TC_00010 were selected for training (20 images of angle $0^{\circ}$ for each texture), while the rest images in OUTex_TC_00010 and all images in OUTex_TC_00012 ('tl84') and OUTex_TC_00012 ('horizon') are utilized for test, respectively.

\subsection{Experimental results}

\subsubsection{Scale parameters}

Denote the proposed method as MS-LPCM $\mathrm{L}_{\mathrm{d}, \mathrm{N}, \mathrm{P}, \mathrm{R}}$. We fixed the parameters $N$ and $P$ as 4 and 8 , and evaluated the effect of $(\mathrm{R}, \mathrm{d})$ on the classification accuracies of MS-LPCM $\mathrm{d}_{\mathrm{d}, \mathrm{N}, \mathrm{P}, \mathrm{R}}$ by setting them as $\{(1,1),(2,1),(3,1)(1,2),(2,2),(3,2),(1,3)$, $(2,3),(3,3)\}$, respectively. The result is shown in Figure 6.

As can be seen from Figure 6, the proposed method MS$\mathrm{LPCM}_{\mathrm{d}, 4,8, \mathrm{R}}$ could get a comparatively higher classification accuracy on $(\mathrm{R}, \mathrm{d})=(2,1)$ than other settings of parameters. This might be due to the compact structure of the neighborhood (Figure 7) from which the LPCM descriptor retrieves the image feature when the parameters $(\mathrm{d}, \mathrm{N}, \mathrm{P}, \mathrm{R})$ are set as $(1,4,8,2)$. It is shown that every blue pixel in Figure 7 are located next to a green pixel, which forms a strong correlation between the LBP codes calculated from these two sets of pixels. Thus the co-occurrence matrix constructed could capture the discriminative information from the image. In addition, we can see from Figure 6 that the classification accuracy of the MSLPCM $_{d, 4,8, R}$ method when $d<R$ is much higher than when $d>$ $\mathrm{R}$. This could be also due to the intersected distribution of the different sets of pixels (for LBP code calculation).

However, since some textures might have special local structures, no certain parameters of the LBP scale (R) and GLCM distance (d) could be suitable for all the textures. So on different database the highest classification accuracy might be achieved with different parameters.

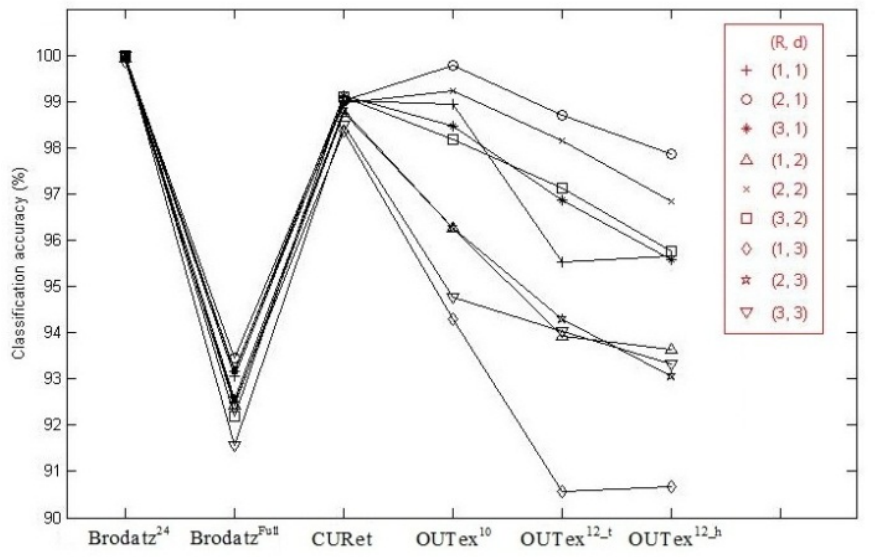

Figure 6 . The classification accuracy on the six datasets by changing the parameters $(\mathrm{R}, \mathrm{d})$ of the LPCM descriptor.

\subsubsection{Comparative evaluation}

In this section, we compare the proposed method with the state-of-the-art approaches introduced in Section 4.1. 


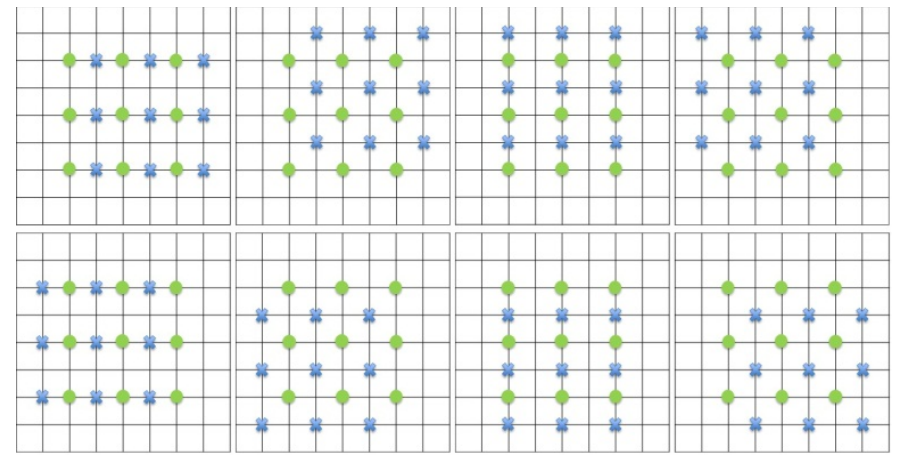

Figure 7. The 8 kinds of pixel distributions from which the $\mathrm{LPCM}_{1,4,8,2}$ descriptor retrieves the feature. The 9 green pixels ('o') and 9 blue pixels (' $x$ ') in each image grid are utilized to get one LBP code respectively.

The $\mathrm{LBP}_{\mathrm{P}, \mathrm{R}}^{\mathrm{riu} 2}$ (including the LBP operator in MS-BP) is implemented in seven scales (or combinations of scales) $\mathrm{LBP}_{8,1}^{\text {riu2 }}, \mathrm{LBP}_{16,2}^{\text {riu2 }}, \mathrm{LBP}_{24,3}^{\text {riu2 }}, \mathrm{LBP}_{8,1+16,2}^{\text {riu2 }}, \mathrm{LBP}_{8,1+24,3}^{\text {riu2 }}$, $\mathrm{LBP}_{16,2+24,3}^{\text {riu } 2} \mathrm{LBP}_{8,1+16,2+24,3}^{\text {riu2 }}$. For VZ_MR8 and VZ_Joint, the number of textons learnt from each class of textures to construct the dictionary is fixed as 10 . The patch size of the VZ Joint method is variable.

In the three datasets - Brodatz $^{24}$, Brodatz ${ }^{\text {Full }}$, and CURet where the training samples are randomly selected, the average classification accuracy of 20 trials is recorded.

The results of the proposed method and the three state-ofthe-art approaches applied on the six datasets are shown in Table 1. From this table it can be seen that the proposed method could get a much higher classification accuracy than the three state-of-the-art approaches, even if the parameters $(\mathrm{d}, \mathrm{N}, \mathrm{P}, \mathrm{R})$ is fixed as $(1,4,8,2)$. And except in Brodatz ${ }^{24}$ and CURet, the MS-LPCM $1,4,8,2$ could get the highest classification accuracy among all the parameter settings.

Furthermore, we also compared the results of MS$\mathrm{LPCM}_{\mathrm{d}, \mathrm{N}, \mathrm{P}, \mathrm{R}}$ method with the best ones found in the literature. As can be seen from Table 2 the proposed method has obtained higher classification accuracy on all the datasets except Brodat ${ }^{\text {Full }}$. The reason might lie in the small number of training samples involved, since in Brodatz ${ }^{24}$ where the number of training samples is 13 we have achieved the highest classification rate. Nonetheless, the authors in [12] used both the Harris and Laplacian detectors to detect the interest areas, and then combined the SIFT and SPIN descriptors to extract feature from each interest area, to achieve the high classification accuracy, which took a large amount of computation.

To prove the efficiency of the chi-square kernel based SVM, we also adopted the linear SVM and some other kernel based SVM for classification to be compared with the chisquare kernel, as shown in TABLE 3 . We could see that the performance of other SVMs is not as good as that of the chisquare kernel, especially for the RBF kernel, because of the difficulties for choosing an appropriate parameter of the RBF.

Other than the classification accuracy, we evaluated the computation complexity of the proposed method as compared with the three state-of-the-art algorithms. We recorded the time for each method to classify a textural image in the CURet dataset on a computer with the $2.70 \mathrm{GHz}$ Intel CPU.

TABLE 4 shows that the proposed method only takes $0.0715 \mathrm{~s}$, which is just a little more than $\operatorname{LBP}_{8,1}^{\text {riu2 }}, \operatorname{LBP}_{16,2}^{\text {riu2 }}$ and $\mathrm{LBP}_{8,1+16,2}^{\text {riu2 }}$, but far more less than the two texton based methods - VZ_MR8 and VZ Joint, where the vector quantization is time consuming.

The success of the MS-LPCM $\mathrm{d}_{\mathrm{d}, \mathrm{N}, \mathrm{P}, \mathrm{R}}$ method largely relies on the representativeness of the LBP method to describe the textures. And since LBP retrieves features from a small local neighborhood, it is enough to use a small-size dictionary. Regarding to the visual words-based co-occurrence method [15], the local patterns were extracted from a large area, thus a small-size dictionary won't be representative enough.

\section{CONCLUSION}

To overcome the drawbacks of existing statistical methods for textural image classification that they not only failed to consider the co-occurrence information, but also were computationally expensive in feature extraction due to the requirement of vector quantization, a multi-scale local pattern co-occurrence matrix (MS-LPCM) descriptor was proposed in this paper to characterize the textural images more effectively and efficiently. Specifically, this textural image classification framework is implemented through four major steps. Firstly, the Gaussian pyramid pre-processing is applied to original textural images to capture multi-scale structural information; secondly, the local binary pattern (LBP) operator is utilized to extract the local patterns from the multiple scaled Gaussian filtered images without the need of vector quantization; thirdly, the gray-level co-occurrence matrix (GLCM) was computed from each scale of LBPs to retrieve both the occurrence and co-occurrence information of those patterns; finally, the texture feature vector cascaded by the lower triangle of the modified LBP based GLCM served as the inputs to a kernel SVM for classification.

The performance of the proposed approach was validated by comparing it with three existing state-of-the art approaches on six datasets which were chosen from three popular databases. Experimental results have demonstrated superior performance of our method. This result would be ascribed to the facts that the multi-scale local binary pattern co-occurrence matrix (MS_LPCM) descriptor is more effective since it can retrieve both the occurrence and the co-occurrence information of different sizes of local patterns (i.e., local neighborhoods). Furthermore, a higher efficiency in both texture feature computation and classification have also been achieved, since more compact feature vectors are resulted and the vector quantization is avoided in the new LBP descriptor.

As a future work, we plan to explore other descriptors which might be more suitable for representing local patterns (neighborhoods) than LBP and to reduce the dimensions of their co-occurrence matrix without losing the discriminative power to achieve more robust and efficient classification of the texture. 
TABLE 1. COMPARISON OF THE CLASSIFICATION ACCURACY (\%) OF THE PROPOSED METHOD AND THE SELECTED APPROACHES

\begin{tabular}{|c|c|c|c|c|c|c|}
\hline & Brodatz $^{24}$ & Brodatz $^{\text {Full }}$ & CURet & OUTex ${ }^{10}$ & OUTex ${ }^{12 \_t}$ & OUTex ${ }^{12 \_h}$ \\
\hline $\mathrm{LBP}_{\mathrm{P}, \mathrm{R}}^{\text {riu2 }}$ & 98.49 & 89.08 & 96.04 & 97.81 & 87.43 & 87.07 \\
\hline VZ_MR8 & 96.60 & 87.76 & 97.79 & 93.59 & 92.55 & 92.82 \\
\hline VZ_Joint & 99.93 & 92.88 & 97.66 & 92.00 & 91.41 & 92.06 \\
\hline MS-BP & 99.37 & 88.85 & 96.16 & 99.30 & 98.26 & 97.08 \\
\hline MS-LPCM $1,4,8,2$ & 99.95 & 93.45 & 99.01 & 99.77 & 98.70 & 97.87 \\
\hline Best of MS-LPCM ${ }_{d, 4,8, R}$ (by changing $(d, R)$ ) & 100.00 & 93.45 & 99.11 & 99.77 & 98.70 & 97.87 \\
\hline
\end{tabular}

TABLE 2. COMPARISON OF OUR RESULTS WITH THE HIGHEST CLASSIFICATION ACCURACY (\%) ACHIEVED SO FAR

\begin{tabular}{|l|l|l|l|l|l|l|}
\hline & Brodatz $^{24}$ & Brodatz $^{\text {Full }}$ & CURet & OUTex $^{10}$ & OUTex $^{12 \_t}$ & OUTex $^{\text {t }}{ }^{\text {h }}$ \\
\hline Best so far & $99.95[25]$ & $95.40[19]$ & $98.52[25]$ & $99.30[24]$ & $98.26[24]$ & $97.08[24]$ \\
\hline MS-LPCM \\
\hline$d, N, P, R$ & 100.00 & 93.45 & 99.11 & 99.77 & 98.70 & 97.87 \\
\hline
\end{tabular}

TABLE 3. COMPARISON OF THE CHI-SQUARE KERNEL WITH THE LINEAR SVM AND OTHER KERNEL BASED SVM.

\begin{tabular}{|l|l|l|l|l|l|l|}
\hline & Brodatz $^{24}$ & Brodatz $^{\text {Full }}$ & CURet & OUTex $^{10}$ & OUTex $^{12 \_}{ }^{\mathrm{t}}$ & OUTex $^{12 \mathrm{~h}}$ \\
\hline Linear SVM & 100 & 90.47 & 98.06 & 95.70 & 93.47 & 91.11 \\
\hline Polynomial & 99.96 & 89.13 & 97.84 & 95.26 & 92.70 & 89.53 \\
\hline RBF & 98.73 & 84.12 & 96.51 & 69.16 & 55.43 & 51.08 \\
\hline
\end{tabular}

TABLE 4. TIME REQUIRED (S) TO RETRIEVE FEATURE FROM A TEXTURAL IMAGE IN THE CURET DATASET

\begin{tabular}{|c|c|c|c|c|}
\hline & $\mathrm{LBP}_{\mathrm{P}, \mathrm{R}}^{\text {riu2 }}$ & VZ_MR8 (11*11 filter) & VZ_Joint ( $3 * 3$ patch) & MS-LPCM $1,4,8,2$ \\
\hline Time (s) & $0.016(8,1), 0.032(16,2), 0.081(24,3)$ & 5.931 & 6.092 & 0.0715 \\
\hline
\end{tabular}

\section{REFERENCE}

[1] M. Varma and A. Zisserman, "A Statistical Approach to Texture Classification from Single Images," International Journal of Computer Vision, vol. 62, pp. 61-81, 2005.

[2] A. Heyden, et al., "Classifying Images of Materials: Achieving Viewpoint and Illumination Independence," in Computer Vision - ECCV 2002. vol. 2352, ed: Springer Berlin / Heidelberg, 2002, pp. 255-271.

[3] R. M. Haralick, "Statistical and structural approaches to texture," Proceedings of the IEEE, vol. 67, pp. 786-804, 1979.

[4] G. Castellano, et al., "Texture analysis of medical images," Clinical Radiology, vol. 59, pp. 1061-1069, 2004.

[5] T. Leung and J. Malik, "Representing and Recognizing the Visual Appearance of Materials using Three-dimensional Textons," Int. J. Comput. Vision, vol. 43, pp. 29-44, 2001.

[6] C. Schmid, "Constructing models for content-based image retrieval," in IEEE Computer Society Conference on Computer Vision and Pattern Recognition, 2001, pp. II-39-II-45 vol.2.

[7] M. Varma and A. Zisserman, "A Statistical Approach to Material Classification Using Image Patch Exemplars," Pattern Analysis and Machine Intelligence, IEEE Transactions on, vol. 31, pp. 2032-2047, 2009.

[8] J. Xie, et al., "Texture classification via patch-based sparse texton learning," in Image Processing (ICIP), 2010 17th IEEE International Conference on, 2010, pp. 2737-2740.

[9] G. Csurka, et al., "Visual categorization with bags of keypoints," in In Workshop on Statistical Learning in Computer Vision, ECCV, 2004, pp. 1-22.

[10] S. Lazebnik, et al., "A sparse texture representation using local affine regions," Pattern Analysis and Machine Intelligence, IEEE Transactions on, vol. 27, pp. 1265-1278, 2005.

[11] K. Mikolajczyk and C. Schmid, "A performance evaluation of local descriptors," Pattern Analysis and Machine Intelligence, IEEE Transactions on, vol. 27, pp. 1615-1630, 2005.

[12] J. Zhang, et al., "Local Features and Kernels for Classification of Texture and Object Categories: A Comprehensive Study," Int. J. Comput. Vision, vol. 73, pp. 213-238, 2007.

[13] D. G. Lowe, "Distinctive Image Features from Scale-Invariant Keypoints," International Journal of Computer Vision, vol. 60, pp. 91-110, 2004.
[14] N. Dalal and B. Triggs, "Histograms of Oriented Gradients for Human Detection," presented at the Proceedings of the 2005 IEEE Computer Society Conference on Computer Vision and Pattern Recognition, 2005.

[15] Y. Yang and S. Newsam, "Spatial Pyramid Co-occurrence for Image Classification," in IEEE International Conference on Computer Vision, 2011.

[16] R. M. Haralick, et al., "Textural Features for Image Classification," IEEE Transactions on Systems, Man and Cybernetics, vol. 3, pp. 610-621, 1973.

[17] T. Ojala, et al., "Multiresolution gray-scale and rotation invariant texture classification with local binary patterns," IEEE Transactions on Pattern Analysis and Machine Intelligence, vol. 24, pp. 971-987, 2002.

[18] B. Boser, et al., "A training algorithm for optimal margin classifiers," in Proceedings of the fifth annual workshop on Computational learning theory, Pittsburgh, Pennsylvania, United States, 1992, pp. 144-152.

[19] J. Zhang, et al., "Local Features and Kernels for Classification of Texture and Object Categories: A Comprehensive Study," International Journal of Computer Vision, vol. 73, pp. 213-238, 2007.

[20] C.-C. Chang and C.-J. Lin, " $\{$ LIBSVM $\}$ : A library for support vector machines," ACM Transactions on Intelligent Systems and Technology, vol. 2, pp. 27:1--27:27, 2011.

[21] P. Brodatz, Textures: A Photographic Album for Artists and Designers: Dover Publications, 1999.

[22] K. J. Dana, et al., "Reflectance and texture of real-world surfaces," ACM Trans. Graph., vol. 18, pp. 1-34, 1999.

[23] T. Ojala, et al., "Outex - New Framework for Empirical Evaluation of Texture Analysis Algorithms," presented at the Proceedings of the 16 th International Conference on Pattern Recognition (ICPR'02) Volume 1 - Volume 1, 2002.

[24] Y. He, et al., "Pyramid-Based Multi-structure Local Binary Pattern for Texture Classification," in Computer Vision - ACCV 2010. vol. 6494, R. Kimmel, et al., Eds., ed: Springer Berlin / Heidelberg, 2011, pp. 133-144.

[25] L. Liu and P. Fieguth, "Texture Classification from Random Features," IEEE Transactions on Pattern Analysis and Machine Intelligence, vol. PP, pp. 1-1, 2011. 